\title{
On the Poincaré series for diagonal forms over algebraic number fields
}

\author{
by \\ Jun WANG (Dalian)
}

1. Introduction. Let $p$ be a fixed prime and $f\left(x_{1}, \ldots, x_{s}\right)$ a polynomial with coefficients in $\mathbb{Z}_{p}$, the $p$-adic integers. Let $c_{n}$ denote the number of solutions of $f=0$ over the ring $\mathbb{Z} / p^{n} \mathbb{Z}$, with $c_{0}=1$. Then the Poincaré series $P_{f}(t)$ is the generating function

$$
P_{f}(t)=\sum_{n=0}^{\infty} c_{n} t^{n}
$$

This series was introduced by Borevich and Shafarevich [1, p. 47], who conjectured that $P_{f}(t)$ is a rational function of $t$ for all polynomials. This was proved by Igusa in 1975 in a more general setting, by using a mixture of analytic and algebraic methods $[5,6]$. Since the proof is nonconstructive, deriving explicit formulas for $P_{f}(t)$ is an interesting problem. In this direction Goldman $[2,3]$ treated strongly nondegenerate forms and algebraic curves all of whose singularities are "locally" of the form $\alpha x^{a}=\beta y^{b}$, while polynomials of form $\sum x_{i}^{d_{i}}$ with $p \nmid d_{i}$ were investigated earlier by E. Stevenson [7], using Jacobi sums. In [8] explicit formulas for $P_{f}(t)$ were derived for diagonal forms. This paper generalizes the results of [8] to algebraic number fields.

Let $F$ be a finite extension of the rational field, and $P$ a prime ideal of $F$ with norm $N(P)=q$ which is a rational prime power. Using the previous notations, we let $c_{n}$ denote the number of solutions of the congruence

$$
a_{1} x_{1}^{d_{1}}+\ldots+a_{s} x_{s}^{d_{s}} \equiv 0\left(\bmod P^{n}\right),
$$

where $d_{1}, \ldots, d_{s}$ are positive integers, $a_{1}, \ldots, a_{s}$ are integers of $F$ prime to $P$, and write $P(t)=\sum_{n=0}^{\infty} c_{n} t^{n}$.

It is clear that $c_{n}=q^{n(s-1)}$ if $d_{i}=1$, for some $i, 1 \leq i \leq s$. Therefore we assume that $d_{1}, \ldots, d_{s}$ are all integers greater than 1 .

Research supported by the National Natural Science Foundation of China. 
Throughout this paper, we set $d=\operatorname{lcm}\left\{d_{1}, \ldots, d_{s}\right\}, f_{i}=d / d_{i}, r=f_{1}+$ $\ldots+f_{s}$ and $\bar{c}_{n}=q^{-n(s-1)} c_{n}$.

2. Exponential sums. For the prime ideal $P$ of $F$, choose an ideal $C$ of $F$ such that $(P, C)=1$ and $P C=(\theta)$ is principal. Then we may assume that any integer $u$ in $F$ is of the form

$$
u=\theta^{j} \xi \quad(j \geq 0,(\xi, P)=1) .
$$

In this case we write $\operatorname{ord}_{P} u=j$. Let $D$ represent the different of $F$ (see $\left[4\right.$, Ch. 36]), and choose $B,(B, P)=1$ such that $(\zeta)=B / P^{n} D$ is principal. We set $\zeta_{m}=\zeta \theta^{n-m}, 0 \leq m \leq n$, such that $\zeta=\zeta_{n}$, and define further

$$
e_{m}(u)=e^{2 \pi i \operatorname{tr}\left(u \zeta_{m}\right)},
$$

where the symbol $\operatorname{tr}(\gamma)$ denotes the trace in $F$. The function $e_{m}(u)$ defines an additive character $\left(\bmod P^{m}\right)$ and has the following simple properties:

$$
\begin{gathered}
e_{0}(u)=1, \quad e_{m}(u)=e_{m}\left(u^{\prime}\right) \quad \text { if } u \equiv u^{\prime}\left(\bmod P^{m}\right), \\
e_{m}\left(u \theta^{j}\right)=e_{m-j}(u) \quad(0 \leq j \leq m), \\
\sum_{z\left(\bmod P^{m}\right)} e_{m}(u z)= \begin{cases}q^{m} & \text { if } u \equiv 0\left(\bmod P^{m}\right), \\
0 & \text { otherwise. }\end{cases}
\end{gathered}
$$

For $k \geq 1$, we define

$$
S_{m}(u, k)=\sum_{z\left(\bmod P^{m}\right)} e_{m}\left(u z^{k}\right), \quad S_{0}(u, k)=1 .
$$

It is clear that if $m \geq j \geq 0$, then

$$
S_{m}\left(u \theta^{j}, k\right)=q^{j} S_{m-j}(u, k) .
$$

The following lemmas are useful in the proof of the main theorem.

Lemma 1. For any positive integer $k$, there is an integer $a \geq k$ such that whenever $m \geq a$, then

$$
S_{m}(u, k)=q^{k-1} S_{m-k}(u, k), \quad(u, P)=1 .
$$

Proof. Suppose $\operatorname{ord}_{P} k=l$. Then take $a$ to be a positive integer which is greater than $k$ and all of $i(l+1) /(i-1), i=2, \ldots, k$. Thus, when $m \geq a$ we have

$$
i(m-l-1) \geq m, \quad i=2, \ldots, k .
$$

From this it follows that $m \geq l+1$ and

$$
\left\{z\left(\bmod P^{m}\right)\right\}=\left\{y+x \theta^{m-l-1} \mid y\left(\bmod P^{m-l-1}\right), x\left(\bmod P^{l+1}\right)\right\} .
$$

Using the binomial theorem and (7) we have

$$
\left(y+x \theta^{m-l-1}\right)^{k} \equiv y^{k}+k y^{k-1} x \theta^{m-l-1}\left(\bmod P^{m}\right),
$$


and

$$
S_{m}(u, k)=\sum_{y\left(\bmod P^{m-l-1}\right)} e_{m}\left(u y^{k}\right) \sum_{x\left(\bmod P^{l+1}\right)} e_{l+1}\left(u k y^{k-1} x\right) .
$$

Since $\operatorname{ord}_{P} k=l$, by (4), the inner sum is 0 unless $y \equiv 0(\bmod P)$, in which case it has the value $q^{l+1}$. Hence we have, by setting $y=y_{1} \theta$, $y_{1}\left(\bmod P^{m-l-2}\right)$,

$$
S_{m}(u, k)=q^{l+1} \sum_{y_{1}\left(\bmod P^{m-l-2}\right)} e_{m-k}\left(u y_{1}^{k}\right)=q^{k-1} S_{m-k}(u, k) .
$$

Let $a(k)$ be the least positive integer such that (6) holds when $m \geq a(k)$, and write

$$
\varrho=\max \left\{a\left(d_{1}\right), \ldots, a\left(d_{s}\right)\right\} .
$$

Lemma 2. Put $T_{m}=q^{-m s} \sum_{\left(v, P^{m}\right)=1} S_{m}\left(v a_{1}, d_{1}\right) \ldots S_{m}\left(v a_{s}, d_{s}\right)$. Then $T_{d+j}=q^{d-r} T_{j}$ for $j \geq \varrho-1$.

Proof. Since $j \geq \varrho-1$ and $d_{i} \geq 2$, we have $d_{i}+j \geq a\left(d_{i}\right)$. By Lemma 1 one gets

$$
S_{d+j}\left(u, d_{i}\right)=q^{f_{i}\left(d_{i}-1\right)} S_{j}\left(u, d_{i}\right), \quad i=1,2, \ldots, s .
$$

Therefore,

$$
\begin{aligned}
T_{d+j} & =q^{-(d+j) s} \sum_{\left(v, P^{d+j}\right)=1} S_{d+j}\left(v a_{1}, d_{1}\right) \ldots S_{d+j}\left(v a_{s}, d_{s}\right) \\
& =q^{-(d+j) s} \sum_{\left(v, P^{d+j}\right)=1} \prod_{i=1}^{s} q^{f_{i}\left(d_{i}-1\right)} S_{j}\left(v a_{i}, d_{i}\right)=q^{d-r} T_{j} .
\end{aligned}
$$

\section{Main results}

Theorem. Let $\varrho$ be as in (8). We have

(i) recursion: for $n \geq \varrho$,

$$
\bar{c}_{n+d}=c+q^{d-r} \bar{c}_{n},
$$

(ii) the Poincaré series is given by

$$
P(t)=\frac{\left(1-q^{s-1} t\right)\left(\sum_{i=0}^{\varrho+d-1} c_{i} t^{i}-q^{d s-r} \sum_{i=0}^{\varrho-1} c^{i} t^{d+i}\right)+c q^{(\varrho+d)(s-1)} t^{\varrho+d}}{\left(1-q^{s-1} t\right)\left(1-q^{d s-r} t^{d}\right)},
$$

where $c=\bar{c}_{\varrho+d-1}-q^{d-r} \bar{c}_{\varrho-1}$ is a constant depending only upon the diagonal form as in (1). 
Proof. (i) From (4) we have

$$
\begin{aligned}
c_{n} & =q^{-n} \sum_{x_{1}, \ldots, x_{s}\left(\bmod P^{n}\right)} \sum_{u\left(\bmod P^{n}\right)} e_{n}\left(u\left(a_{1} x_{1}^{d_{1}}+\ldots+a_{s} x_{s}^{d_{s}}\right)\right) \\
& =q^{-n} \sum_{u\left(\bmod P^{n}\right)} S_{n}\left(u a_{1}, d_{1}\right) \ldots S_{n}\left(u a_{s}, d_{s}\right) .
\end{aligned}
$$

In the summation over $u\left(\bmod P^{n}\right)$, we may set $u=v \theta^{n-m}, 0 \leq m \leq n$, $v\left(\bmod P^{m}\right)$ and $\left(v, P^{m}\right)=1$. From $(5)$ one has

$$
\begin{aligned}
c_{n} & =q^{n(s-1)} \sum_{m=0}^{n} q^{-m s} \sum_{\left(v, P^{m}\right)=1} S_{m}\left(v a_{1}, d_{1}\right) \ldots S_{m}\left(v a_{s}, d_{s}\right) \\
& =q^{n(s-1)} \sum_{m=0}^{n} T_{m} .
\end{aligned}
$$

Set $n=\varrho+l, l \geq 0$. By Lemma 2, we have

$$
\begin{aligned}
\bar{c}_{n+d} & =\sum_{m=0}^{n+d} T_{m}=\sum_{m=0}^{\varrho+d-1} T_{m}+\sum_{m=0}^{l} T_{\varrho+d+m}=\bar{c}_{\varrho+d-1}+\sum_{m=0}^{l} q^{d-r} T_{\varrho+m} \\
& =\bar{c}_{\varrho+d-1}+q^{d-r}\left(\bar{c}_{n}-\bar{c}_{\varrho-1}\right)=c+q^{d-r} \bar{c}_{n} .
\end{aligned}
$$

(ii) Put $q^{s-1} t=t_{1}$. Then

$$
\begin{aligned}
P(t) & =\sum_{n=0}^{\infty} c_{n} t^{n}=\sum_{i=0}^{\varrho+d-1} c_{i} t^{i}+\sum_{n=\varrho}^{\infty} c_{n+d} t^{n+d} \\
& =\sum_{i=0}^{\varrho+d-1} c_{i} t^{i}+\sum_{n=\varrho}^{\infty} \bar{c}_{n+d} t_{1}^{n+d}=\sum_{i=0}^{\varrho+d-1} c_{i} t^{i}+\sum_{n=\varrho}^{\infty}\left(c+q^{d-r} \bar{c}_{n}\right) t_{1}^{n+d} \\
& =\sum_{i=0}^{\varrho+d-1} c_{i} t^{i}+c t_{1}^{\varrho+d}\left(1-t_{1}\right)^{-1}+q^{d-r} t_{1}^{d}\left(P(t)-\sum_{i=0}^{\varrho-1} c_{i} t^{i}\right) .
\end{aligned}
$$

This gives the result of the theorem.

\section{References}

[1] Z. I. Borevich and I. R. Shafarevich, Number Theory, Academic Press, New York 1966.

[2] J. R. Goldman, Numbers of solutions of congruences: Poincaré series for strongly nondegenerate forms, Proc. Amer. Math. Soc. 87 (1983), 586-590.

[3] -, Numbers of solutions of congruences: Poincaré series for algebraic curves, Adv. in Math. 62 (1986), 68-83.

[4] E. Hecke, Lectures on the Theory of Algebraic Numbers, Springer, New York 1981. 
[5] J. Igusa, Complex powers and asymptotic expansions, II, J. Reine Angew. Math. 278/279 (1979), 307-321.

[6] -, Some observations on higher degree characters, Amer. J. Math. 99 (1977), 393417.

[7] E. Stevenson, The rationality of the Poincaré series of a diagonal form, Thesis, Princeton University, 1978.

[8] J. Wang, On the Poincaré series for diagonal forms, Proc. Amer. Math. Soc., to appear.

INSTITUTE OF MATHEMATICAL SCIENCES

DALIAN UNIVERSITY OF TECHNOLOGY

DALIAN 116024, PEOPLE'S REPUBLIC OF CHINA 